\title{
On the stabilization of neoclassical magnetohydrodynamic tearing modes using localized current drive or heating
}

\author{
C. C. Hegna and J. D. Callen \\ Departments of Nuclear Engineering and Engineering Physics and Physics, University of Wisconsin, \\ Madison, Wisconsin 53706-1687
}

(Received 14 January 1997; accepted 13 May 1997)

\begin{abstract}
The effectiveness of using localized current drive or heating to suppress the formation and growth of neoclassical magnetohydrodynamic (MHD) tearing modes is addressed. The most efficient way to use an auxiliary current source is to cause current to flow in the same direction as the equilibrium bootstrap current and phase the current relative to the magnetic island such that the current is deposited on the O-point of the island. Theoretical estimates for the amount of required current to suppress the formation of a large magnetic island is of order a few percent of the equilibrium current. If the suppression is successful, the magnetic island will saturate at a width of order the radial localization width of the current source. Localized heating at the O-point of the magnetic island can also produce stabilizing effects relative to magnetic island growth. The effects of the driven current or heating can be illustrated by using a phase diagram of the island growth. (C) 1997 American Institute of Physics. [S1070-664X(97)02508-1]
\end{abstract}

\section{INTRODUCTION}

Neoclassical effects modify the resistive magnetohydrodynamic (MHD) description of tearing modes in tokamaks. ${ }^{1-4}$ In particular, the self-consistent effects of perturbed bootstrap currents allow pressure-induced magnetic island formation in the positive shear regions of tokamaks. This instability process can, in principle, give rise to magnetic islands at every low-order rational surface despite the fact that resistive MHD theories predict stability. ${ }^{3,4}$ As tokamak discharges start to operate more routinely at low collisionality, with high $\beta_{\theta}$, large bootstrap fraction and long pulse lengths, neoclassical tearing modes will play a more prominent role in limiting stored energy and reducing confinement.

A number of tokamak experiments have observed the appearance of neoclassical MHD tearing modes. ${ }^{5-9}$ Associated with these modes is the formation of slowly growing magnetic islands that reduce the confinement time of the plasma ${ }^{10}$ or, if the islands grow large enough, cause a disruption. Additionally, in long pulse, low collisionality tokamaks, the neoclassical MHD tearing modes provide beta limits well below that predicted by ideal MHD stability theory. It is anticipated that unless they are stabilized, neoclassical tearing modes will provide a dangerous instability for the proposed International Thermonuclear Experimental Reactor (ITER) device. ${ }^{8}$

In this work, the effect of localized current drive or heating sources on magnetic island formation is addressed. Specifically, we attempt to quantify to what extent these methods can be used to stabilize the neoclassical-MHD tearing mode. A number of proposals have been suggested and studied relative to stabilizing magnetic island formation in tokamaks which are resistive MHD unstable. In particular, these methods include using applied resonant field errors with negative feedback, ${ }^{11-15}$ local heating of the island region, ${ }^{16-23}$ and local current drive mechanisms. ${ }^{24-28} \mathrm{We}$ extend these studies and use a similar procedure to study the local heating and current drive effects on neoclassical MHD tearing modes.

An analytic theory can be developed by including the effects of perturbed helical bootstrap currents, and the localized sources through a generalized Ohm's law. In Sec. III a nonlinear island evolution equation is derived that includes the effects of a phase localized current source. The stability properties with this new term are illustrated through the use of phase diagrams for the island evolution. In Sec. IV, the effects of localized heating are addressed. Localized heating gives rise to self-consistent deformations of the current profile through the temperature dependency of the plasma resistivity. Finally, we apply these results to indicate the level needed to suppress the formation of large amplitude neoclassical MHD tearing modes.

\section{ISLAND EVOLUTION EQUATION}

Consider an equilibrium with a monotonically increasing $q$ profile and magnetic surfaces labeled by the "radial" coordinate $\rho$. The poloidal flux function $\psi$ is a function of $\rho, \psi=\psi(\rho)$. The equilibrium magnetic field is written $B_{0}$ $=I(\rho) \nabla \zeta+\psi^{\prime} \nabla \zeta \times \nabla \rho=q(\rho) \psi^{\prime} \nabla \rho \times \nabla \theta+\psi^{\prime} \nabla \zeta \times \nabla \rho$ where $\psi^{\prime}$ $=d \psi / d \rho$. In this work, a small aspect ratio assumption is used, so that $|B| \cong I|\nabla \zeta|=I / R$ where $R$ is the major radius.

Magnetic reconnection can occur at the rational surface $q\left(\rho_{s}\right)=m / n$. Now consider a magnetic perturbation resonant at that surface $\rho=\rho_{s}$ of the form $\mathbf{B}_{1}=\nabla \zeta \times \nabla \widetilde{\psi}$, where $\widetilde{\psi}$ $=\psi_{1}(\rho) \cos (m \theta-n \zeta)$. Near the rational surface, the total magnetic field has the approximate form, $\mathbf{B} \cong q_{s} \psi_{s}^{\prime} \nabla \rho \times \nabla \alpha$ $-\left(\psi_{s}^{\prime} q_{s}^{\prime} / q_{s}\right) \nabla \zeta \times \nabla \Psi$, where

$$
\Psi=\frac{1}{2} x^{2}-\frac{q \widetilde{\psi}}{q^{\prime} \psi^{\prime}},
$$

$\alpha=\theta-\zeta / q_{s}$ is the resonant angle, $x=\rho-\rho_{s}$ is the distance away from the rational surface, $q_{s}=q\left(\rho_{s}\right), q_{s}^{\prime}=q^{\prime}\left(\rho_{s}\right)$, and $\psi_{s}^{\prime}=\psi^{\prime}\left(\rho_{s}\right)$. In the vicinity of the island region, $\mathbf{B} \cdot \nabla \Psi$ $=0$, indicating that $\Psi$ labels the perturbed magnetic flux surfaces. 
Using the constant- $\psi$ assumption of tearing mode theory $\psi_{1}(\rho) \cong \psi_{1}\left(\rho_{s}\right)$, the perturbation gives rise to an island of full width $W$

$$
W=4 \sqrt{\frac{q_{s} \psi_{1}}{q_{s}^{\prime} \psi_{s}^{\prime}}} .
$$

The local approximations are valid if the small island assumption $W<\rho_{s}$ is satisfied. A helical flux surface averaging operator is defined by

$$
\langle C\rangle=\frac{\oint \frac{d \alpha}{2 \pi} \frac{C}{\sqrt{2 \Psi+w^{2} \cos (m \alpha) / 8}}}{\oint \frac{d \alpha}{2 \pi} \frac{1}{\sqrt{2 \Psi+w^{2} \cos (m \alpha) / 8}}}
$$

for any function $C$. It has the property $\mathbf{B} \cdot \nabla\langle C\rangle=0$.

In the relevant nonlinear regime where the island width exceeds the linear layer width, a variant on Rutherford theory is used to construct an island evolution equation. ${ }^{29} \mathrm{In}$ this asymptotic theory, the form of the current in the vicinity of the rational surface is calculated and matched to the exterior matching parameter $\Delta^{\prime}$, the logarithmic jump in the vector potential across the magnetic surface as constructed in the exterior region. Specifically, the matching condition is

$$
\frac{1}{2} \Delta^{\prime} \psi_{1}=\mu_{0} R \int_{-\infty}^{\infty} d \rho \oint \frac{d \alpha}{2 \pi} \cos (m \alpha) J_{\|} .
$$

In the limit that curvature and inertial effects can be neglected, the current in the vicinity of the island satisfies $\mathbf{B}$ - $\nabla J_{\|}=0$. This condition is met by demanding that the current profile be a function of the flux surface, $J_{\|}=J_{\|}(\Psi)$ where $\Psi$ is given in Eq. (1).

The description of the inner region solution is completed by introducing an Ohm's law that self-consistently accounts for the island in the local magnetic topology. The flux surface averaged parallel current is given by

$$
J_{\|}(\Psi)=\frac{1}{\eta}\left\langle E_{\|}\right\rangle+\frac{1}{\eta B}\left\langle\mathbf{B} \cdot \nabla \cdot \pi_{\| e}\right\rangle+\left\langle J_{\text {aux }}\right\rangle,
$$

where the first term is the inductive electric field which develops due to the time dependent magnetic island growth. Specifically, this is given by

$$
\left\langle E_{\|}\right\rangle=\frac{1}{R} \frac{d \psi_{1}}{d t}\langle\cos (m \alpha)\rangle .
$$

The last term in Eq. (5) allows for an externally controlled driven magnetic current. Particular forms for $J_{\text {aux }}$ will be considered in the following sections. The second term describes the neoclassical effects which give rise to the perturbed bootstrap current. It can shown that the form for this term is given by ${ }^{1-4,30-32}$

$$
\left\langle\mathbf{B} \cdot \nabla \cdot \pi_{\| e}\right\rangle=-\frac{\mu_{e}}{\nu_{e}} \frac{I}{\psi_{s}^{\prime}}\left\langle\frac{\partial p}{d x}\right\rangle,
$$

where $\mu_{e}$ is the electron viscous damping rate and $\nu_{e}$ is the electron collision frequency. For sufficiently large magnetic islands, ${ }^{30-32}$ the pressure profile in the vicinity of the rational surface is also a perturbed flux function, $p=p(\Psi)$, which implies $\langle\partial p / \partial x\rangle=\langle x\rangle d p / d \Psi$. Assuming a cross-field diffusion process and a pressure source located away from the rational surface, the pressure profile in the vicinity of the rational surface is given by ${ }^{1-4,30,33}$

$$
\frac{d p}{d \Psi}=\left.\frac{d p}{d \rho}\right|_{\text {eq }} \frac{\Theta\left(\Psi-W^{2} / 16\right)}{\oint \frac{d \alpha}{2 \pi} \sqrt{2 \Psi+W^{2} \cos \alpha / 8}},
$$

where $\Theta$ is a step function and $d p /\left.d \rho\right|_{\mathrm{eq}}=p_{s}^{\prime}$ is the equilibrium value of the pressure gradient in the absence of the magnetic island. This model of the pressure profile has a flat spot inside the island separatrix. For island widths smaller than a characteristic distance determined by the ratio of perpendicular to parallel equilibration processes, the pressure is not a flux function and a different treatment is needed. For the purposes of this paper, we will restrict our calculation to be relevant for island widths larger than any characteristic threshold distances. ${ }^{30-32,34,35}$

Using Eqs. (5)-(8) in the matching condition, Eq. (4), leads to an island evolution equation for $w$ assuming $J_{\text {aux }}$ $=0$ and that the island width exceeds the threshold condition, ${ }^{1-4}$

$$
0.82 \frac{d w}{d t}=\frac{1}{\tau_{r}}\left(\Delta^{\prime} \rho_{s}+\frac{D_{n c}}{w}\right),
$$

where $w=W / \rho_{s}$ is the island width normalized to the local minor radius, $\tau_{r}=\mu_{0} \rho_{s}^{2} / \eta$, and

$$
D_{n c}=-4.6 \sqrt{\epsilon} \frac{2 \mu_{0} p_{s}^{\prime} R^{2}}{\psi_{s}^{\prime 2}} \frac{q_{s}}{q_{s}^{\prime}}
$$

describes the effect of the perturbed bootstrap current and the large aspect ratio, small collisionality form for $\mu_{e}$ is used where $\epsilon=\rho_{s} / R$ is the local aspect ratio. It should be pointed out that additional effects due to polarization currents ${ }^{34,35}$ and curvature ${ }^{36}$ add terms to Eq. (9) and finite pressure diffusion properties modify the neoclassical and curvature terms. ${ }^{30-32}$ However, these terms only play a role at small island width where they influence the nonlinear island threshold condition. These terms play very little role in determining the evolution properties of the mode above the threshold or the saturated island width.

Since $q^{\prime}$ is generally positive for tokamak discharges, the neoclassical term in Eq. (9) is destabilizing and can cause the formation of magnetic islands even when $\Delta^{\prime}$ is negative and stabilizing. When $\Delta^{\prime}<0$, the islands grow and saturate at the level

$$
w_{\mathrm{sat}}=\frac{D_{n c}}{-\Delta^{\prime} \rho_{s}}
$$

which corresponds to island widths of typical size, $W_{\text {sat }}$ $\cong \rho_{s} \downarrow \epsilon \beta_{\theta} / m$ which can be a significant fraction of $\rho_{s}$. In this work, we will concentrate on the suppression the bootstrap current driven magnetic island in the $\Delta^{\prime}<0$ limit. However, the treatment given here can be easily extended to treat the $\Delta^{\prime}>0$ case. 


\section{LOCAL CURRENT DRIVE STABILIZATION}

In this section we include the effect of an auxiliary current drive source in the island region. In order to produce a stabilizing effect, the additional current source is required to deliver current in the same direction as the bootstrap current, provided that the source is localized in phase to the O-point of the island. Physically, this can be understood by noting that the origin of the instability mechanism of the neoclassical MHD tearing mode is the absence of bootstrap current inside the island separatrix. ${ }^{4}$ The intention of using a localized source is to replace this current. ${ }^{28}$ Following the procedure of the previous section, an island evolution equation can be derived from Eqs. (4) and (5) in the form

$$
0.82 \frac{d w}{d t}=\frac{1}{\tau_{r}}\left(\Delta^{\prime} \rho_{s}+\frac{D_{n c}}{w}-\frac{D_{\text {aux }}}{w^{2}} \eta_{\text {aux }}\right),
$$

where $D_{\text {aux }}$ is dimensionless and is given by

$$
D_{\text {aux }}=\frac{I_{\text {aux }} \mu_{0} R}{s \psi_{s}^{\prime} \rho_{s}} \frac{16}{\pi},
$$

$s=\rho q_{s}^{\prime} / q_{s}$ is the magnetic shear,

$$
I_{\text {aux }}=2 \pi \rho \int d \rho \oint \frac{d \alpha}{2 \pi}\left\langle J_{\text {aux }}\right\rangle
$$

is the total amount of auxiliary current driven in the island region, and

$$
\eta_{\text {aux }}=\frac{\int d \rho \oint \frac{d \alpha}{2 \pi} \cos (m \alpha)\left\langle J_{\text {aux }}\right\rangle}{\int d \rho \oint \frac{d \alpha}{2 \pi}\left\langle J_{\text {aux }}\right\rangle}
$$

is a measure of how efficient the helical current localization procedure is. In the ideal limit of a $\delta$-function current source on the O-point of the magnetic island $(\alpha=0), \eta_{\text {aux }}=1$. Note the quantity evaluated in Eqs. (14) and (15) is the auxiliary current averaged over the modified flux surface. This averaging denotes the fast equilibration of the current along field lines as described in the leading order quasineutrality condition. Since $\left\langle J_{\text {aux }}\right\rangle$ is a flux function, $\eta_{\text {aux }}$ can be written

$$
\eta_{\text {aux }}=\frac{\int_{-1}^{\infty} d \Omega\left\langle J_{\text {aux }}\right\rangle \oint d \alpha \cos (m \alpha)[\Omega+\cos (m \alpha)]^{-1 / 2}}{\int_{-1}^{\infty} d \Omega\left\langle J_{\text {aux }}\right\rangle \oint d \alpha[\Omega+\cos (m \alpha)]^{-1 / 2}},
$$

where $\Omega=16 \Psi / W^{2}$ is a normalized flux surface label where $\Omega=-1$ is the island O-point and $\Omega=1$ is the separatrix.

A simple model for the auxiliary current profile is a constant current density located inside a particular flux surface $\Omega_{J},\left\langle J_{\text {aux }}\right\rangle \sim \Theta\left(\Omega_{J}-\Omega\right)$. The parameter $\Omega_{J}$ can be interpreted as $\Omega_{J}=2 \delta_{J}^{2} / W^{2}-1$ where $\delta_{J}$ is the radial localization distance across the O-point region $(\alpha=0)$ (see Fig. 1). For this model of $\left\langle J_{\text {aux }}\right\rangle, \eta_{\text {aux }}$ is given by

$$
\eta_{\text {aux }}=1-\frac{2}{3} \frac{\left(2-k^{2}\right) E(k)+\left(2 k^{2}-2\right) K(k)}{E(k)-\left(1-k^{2}\right) K(k)},
$$

where $k=\delta_{J} / W$, and $K(k)$ and $E(k)$ are complete elliptic integrals of the first and second kind. Figure 2 shows a plot for $\eta_{\text {aux }}$ as a function of $\delta_{J} / W$. It demonstrates that for

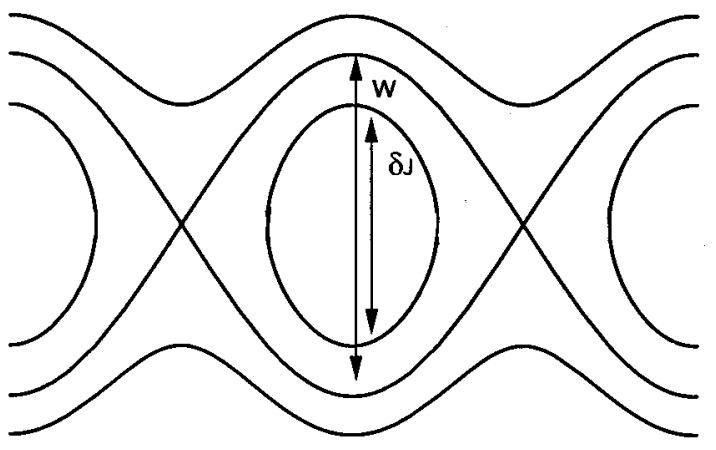

FIG. 1. The magnetic island topology in $x-\alpha$ space. The characteristic distances for the island width $w$ and current localization $\delta_{J}$ are shown.

$\delta_{J} / W \ll 1, \eta_{\text {aux }}$ does not vary greatly, but as the localization width approaches the island width, $\eta_{\text {aux }}$ can be reduced substantially.

As $\delta_{J}$ is increased to much larger than the island width, the efficiency function $\eta_{\text {aux }}$ as derived for the step function model drops quickly. In the asymptotic limit $\delta_{J} \gg W, \eta_{\text {aux }}$ $\cong W^{2} / 8 \delta_{J}^{2}$ and Eq. (12) takes the form

$$
0.82 \frac{d w}{d t} \cong \frac{1}{\tau_{r}}\left(\Delta^{\prime} \rho_{s}+\frac{D_{n c}}{w}-D_{\text {aux }} \frac{\rho_{s}^{2}}{8 \delta_{J}^{2}}\right)
$$

where the last term is independent of $w$.

The stability properties of the neoclassical tearing mode can be described by the phase space diagram of the magnetic island (see Fig. 3). For the purposes of this plot, it is assumed that $\Delta^{\prime}<0, D_{n c}>0$, and $D_{\text {aux }}>0$ so that the bootstrap current term is destabilizing and the added current is stabilizing. Additionally, for the purposes of simplifying the analysis, we will assume $W_{\text {sat }}=D_{n c} /\left(-\Delta^{\prime}\right) \gg \delta_{J}$, the saturated island width in the $D_{\text {aux }}=0$ limit is larger than the current localization width. In Fig. 3, the island width is normalized to $W_{\text {sat }}$ and $\delta_{J} / W_{\text {sat }}=0.1$.

As described by Eqs. (12) and (18) and shown in Fig. 3, there are an odd number of steady solutions to the island evolution equation. At a sufficiently small enough value of $D_{\text {aux }}$, there is only one steady solution to Eq. (12) given by

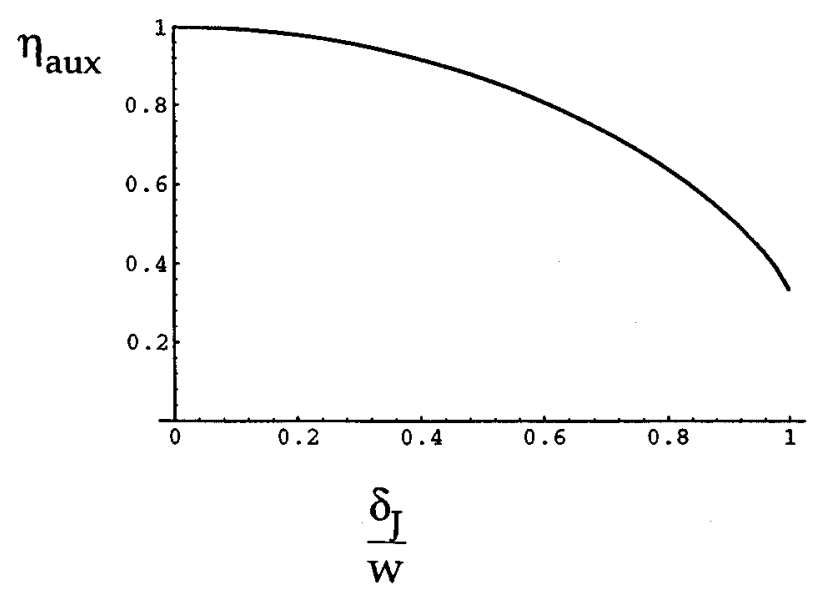

FIG. 2. The efficiency function $\eta_{\text {aux }}$ given by Eq. (17) is plotted as a function of $\delta_{J} / W$. As this ratio becomes small, $\eta_{\text {aux }}$ stays close to unity. 


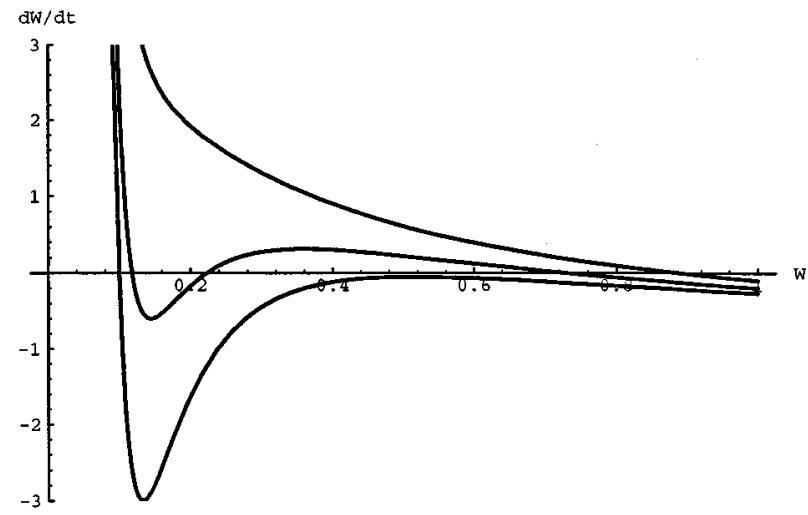

FIG. 3. The effect of the phased auxiliary current drive is demonstrated with a phase space diagram of the magnetic island dynamics as described by Eq. (12). The assumption $\Delta^{\prime}<0$ is made, where the island width is normalized to the saturated value $W_{\text {sat }}=w_{n c}^{0} /\left(-\Delta^{\prime}\right)$ and $\delta_{J}=0.1 W_{\text {sat }}$. The top curve is the phase space of the island evolution for $w_{\text {aux }}<4 \delta_{J} D_{n c} / \rho_{s} \sqrt{3}$. In this limit the auxiliary current has very little effect on the island evolution and saturation. The middle curve demonstrates typical solutions for $4 \delta_{J} D_{n c} / \rho_{s} \sqrt{3}$ $<D_{\text {aux }}<D_{n c} w_{\text {sat }} / 4 \eta_{\text {aux }}$. For this case there are two stable fixed point solutions given by Eqs. (19) and (22), and one unstable fixed point solution. The lowest curve is the phase space evolution for $D_{\text {aux }}>D_{n c}^{0} w_{\text {sat }} / 4 \eta_{\text {aux }}$. In this limit, only the small saturated island width solution is a fixed point.

$$
w_{\text {fixed }, J 1}=\frac{D_{n c}}{\left(-\Delta^{\prime} \rho_{s}\right)}\left\{\frac{1}{2}+\sqrt{\frac{1}{4}-\frac{\eta_{\text {aux }} D_{\text {aux }}\left(-\Delta^{\prime} \rho_{s}\right)}{\left(D_{n c}\right)^{2}}}\right\},
$$

where in the limit $\eta_{\text {aux }} D_{\text {aux }} \ll\left(D_{n c}\right)^{2} /\left(-\Delta^{\prime} \rho_{s}\right), w_{\text {fixed, } J 1}$ $\cong w_{\text {sat }}$. In this case, the auxiliary current is very small and the island evolution is essentially unaffected in that the only fixed point solution is that given by Eq. (11). A characteristic solution of this form is shown as the top curve of the phase diagram.

At larger values of $D_{\text {aux }}$, two additional fixed point solutions appear. By using an analytic approximation to $\eta_{\text {aux }}$ $\cong 1-2 \delta_{J}^{2} / 3 W^{2}$, for $W>\delta_{J}$, this transition occurs once the inequality

$$
D_{\text {aux }}>\frac{4}{\sqrt{3}} \frac{\delta_{J}}{\rho_{s}} D_{n c},
$$

is satisfied. The steady solution corresponding to the largest saturated island width is given by $w_{\text {fixed,J1 }}$ of Eq. (19). This is a stable fixed point. The value

$$
w_{\text {fixed }, J 2} \cong \frac{D_{n c}}{\left(-\Delta^{\prime} \rho_{s}\right)}\left\{\frac{1}{2}-\sqrt{\frac{1}{4}-\frac{\eta_{\text {aux }} D_{\text {aux }}\left(-\Delta^{\prime} \rho_{s}\right)}{\left(D_{n c}\right)^{2}}}\right\}
$$

corresponds to an unstable fixed point solution. Equation (21) is valid provided that $w_{\text {fixed, } J 2}>\delta_{J} / \rho_{s}$. The smallest steady solution is approximately given by

$$
w_{\text {fixed }, J 3} \cong \frac{D_{n c}}{\left(-\Delta^{\prime} \rho_{s}\right)+D_{\text {aux }} \rho_{s}^{2} / 8 \delta_{J}^{2}} \cong \frac{8 \delta_{J}^{2} D_{n c}}{D_{\text {aux }} \rho_{s}^{2}},
$$

which is obtained from Eq. (18). In this limit, the saturated island width is of order $\delta_{J}$. Specifically, $w_{\text {fixed,J3 }}$ corresponds to

$$
W_{\text {fixed,J3 }} \sim \delta_{J} \frac{J_{\text {bootstrap }}}{J_{\text {auxiliary }}},
$$

which is the current localization width times the ratio of the local bootstrap to auxiliary current density.

The novel feature of this class of solutions, as described by the middle curve of Fig. 3, is that the steady value $w_{\text {fixed,J2 }}$ takes on the role of a threshold island width condition. For an island width that is initially larger than this value, the island amplitude will grow and saturate at the "large", value given in Eq. (19). However, for island widths which are initially smaller than $w_{\text {fixed, } J 2}$, the island evolution equation indicates an island that will decay down to the "small" saturated value described by Eq. (22).

As the auxiliary current term increases further, the two fixed point solutions $w_{\text {fixed,J1 }}$ and $w_{\text {fixed,J2 }}$ approach each other until they obtain a common value when $\eta_{\text {aux }} D_{\text {aux }}$ $=D_{n c} w_{\text {sat }} / 4$. For

$$
\eta_{\text {aux }} D_{\text {aux }}>\frac{1}{4} \frac{\left(D_{n c}\right)^{2}}{\left(-\Delta^{\prime} \rho_{s}\right)},
$$

there is only one steady solution to the island evolution equation corresponding to the small saturated island width given by Eq. (22). Equation (24) is the condition for the elimination of the large saturated neoclassical tearing mode by auxiliary current drive. The bottom curve of Fig. 3 demonstrates the structure of this solution.

\section{THE EFFECT OF LOCAL HEATING ON NEOCLASSICAL TEARING MODES}

The effect of a local heating source on a neoclassical tearing mode has been discussed previously. ${ }^{33}$ The essential idea behind using localized heating to affect island physics is through the helically resonant temperature variations near an island causing variations in the Ohmic current profile

$$
\delta J_{\|}=\frac{3}{2} \frac{\delta T_{e}}{T_{e o}} J_{\| o},
$$

where $J_{\| o}=E_{\|} / \eta_{o}$ is created from the externally applied loop voltage, $\eta_{o}$ is the resistivity evaluated at $T_{e o}$, and $\delta T_{e}$ is the temperature variation. As can be shown from radial parity considerations, the only contribution from this mechanism comes from temperature perturbations inside the island separatrix.

The temperature profile can be solved ${ }^{33}$ for by assuming a model diffusion equation

$$
\nabla n \chi_{\perp} \nabla T_{e}=-S
$$

where $\chi_{\perp}$ is the cross-field diffusivity and $S$ is the local heating source. For the purposes of this study, it is assumed that on the time scales of interest $T_{e}$ will equilibrate along the perturbed field lines, so that $T_{e}=T_{e}(\Omega)$ where $\Omega$ $=16 \Psi / W^{2}$ is the normalized flux surface label. It is straightforward to show using this assumption that the temperature profile inside the island separatrix is ${ }^{33}$

$$
\frac{d T_{e}}{d \Omega}=-\frac{W^{2}}{32} \frac{\int_{-1}^{\Omega} d \Omega^{\prime} \oint d \alpha \frac{S(\Omega, \alpha)}{\sqrt{\Omega^{\prime}+\cos (m \alpha)}}}{\oint d \alpha n \chi_{\perp} \sqrt{\Omega^{\prime}+\cos (m \alpha)}},
$$


where the boundary condition is $d T_{e} / d \Omega=0$ on the magnetic axis of the island. Equation (27) can be simplified by assuming a uniform $n \chi_{\perp}$ and by using a step function heating profile $S(\Omega)=S_{o} \Theta\left(\Omega_{c}-\Omega\right)$. Using these assumptions,

$$
\begin{aligned}
\frac{d T_{e}}{d \Omega} & =-\frac{W^{2}}{16} \frac{S_{o}}{n \chi_{\perp}} \quad \text { for } \Omega<\Omega_{c}, \\
& =-\frac{W^{2}}{16} \frac{S_{o}}{n \chi_{\perp}} \frac{\oint \sqrt{\Omega_{c}+\cos (m \alpha)}}{\oint \sqrt{\Omega+\cos (m \alpha)}}, \quad \text { for } \Omega_{c}<\Omega<1 .
\end{aligned}
$$

From Eq. (26), the characteristic temperature perturbation size scales as $\delta T_{e} \cong W^{2} S_{o} / 8 n \chi_{\perp}$ for $\Omega_{c}>1$ and $\delta T_{e}$ $\cong \delta_{H} W S_{o} / 8 n \chi_{\perp}$ for $\Omega_{c}<1$ where $\delta_{H}$ is the radial localization of the heating profile defined by $\Omega_{c}=2 \delta_{H}^{2} / W^{2}-1$.

To account for the effect of the local heating on the neoclassical MHD tearing mode, the same procedure as the previous two sections is used. Using Eqs. (25) and (28) in Eq. (4), an island evolution equation is derived. First, for the case of heating throughout the island region, $\Omega_{c}>1$, the evolution equation has the form

$$
0.82 \frac{d w}{d t}=\frac{1}{\tau_{r}}\left(\Delta^{\prime} \rho_{s}+\frac{D_{n c}}{w}-w D_{\text {heat }}\right),
$$

where

$$
D_{\text {heat }}=\frac{16}{5 \pi} \frac{q_{s}}{q_{s}^{\prime}} \frac{R \mu_{o} J_{\| o}}{\psi_{s}^{\prime}} \frac{S_{o} \rho_{s}^{2}}{n T_{e} \chi_{\perp}}
$$

is dimensionless. There is only one fixed point solution of Eq. (29) for $\Delta^{\prime}<0$ and $D_{\text {heat }}>0$. The saturated island width is given by

$$
w_{\mathrm{sat}, H}=\frac{D_{n c}}{-\Delta^{\prime} \rho_{s}} \frac{2}{1+\sqrt{1+\mathrm{Y}}},
$$

where

$$
\Upsilon=4 \frac{D_{\text {heat }} D_{n c}}{\left(-\Delta^{\prime} \rho_{s}\right)^{2}}
$$

is a measure of the effect of the localized heating. In this limit, the local heating can reduce the saturated island width, but cannot completely eliminate the neoclassical tearing mode.

In the limit that the heating width is smaller than the island with $\Omega_{c}<1$, the island evolution equation has the form

$$
0.82 \frac{d w}{d t}=\frac{1}{\tau_{r}}\left(\Delta^{\prime} \rho_{s}+\frac{D_{n c}}{w}-D_{\text {heat }} C_{o} \frac{\delta_{H}}{\rho_{s}}\right),
$$

where $C_{o}$ is a constant of order unity and a weak function of $\delta_{H} / w .{ }^{19}$ The additional heating cannot prevent the mode from growing. However, it does lower the saturated island width. In this limit, the saturated island width is

$$
w_{\text {sat }, H 1}=\frac{D_{n c}^{0}}{-\Delta^{\prime} \rho_{s}+D_{\text {heat }} C_{o} \delta_{H} / \rho_{s}} .
$$

\section{DISCUSSION}

Of the methods discussed in this work, the most effective means of suppressing neoclassical MHD tearing modes is by using a localized current drive source. The driven current should be directed in the same direction as the equilibrium bootstrap current and is most effective if the current is localized to the O-point of the island and has a radial localization width smaller than the characteristic island size. In this limit, it is possible to suppress the existence of a large stable steady state value of a saturated magnetic island. This criterion is given in Eq. (24). This condition can be rewritten

$$
\frac{I_{\text {aux }}}{I_{\text {tor }}} \eta_{\text {aux }} \geqslant 0.08 \frac{\epsilon \beta_{\theta}^{2}}{s m}\left(\frac{\rho}{L_{p}}\right)^{2},
$$

where $I_{\text {tor }}=2 \pi \rho B_{\theta}\left(\rho_{s}\right)$ is the total amount of equilibrium toroidal current flowing inside the rational surface, $L_{p}$ $=-p /(d p / d \rho)$ is the equilibrium length scale of the pressure gradient, $\beta_{\theta}=2 \mu_{o} p / B_{\theta}^{2}$ is the local poloidal beta and the approximation $\Delta^{\prime} \rho_{s}=-2 m$ is used where $m$ is the poloidal mode number. The efficiency factor is assumed to be of order unity and is given by Eq. (17) for the step function model for the auxiliary current drive source. For $\epsilon=1 / 4, m=2$, $\beta_{\theta}=1, s=1$, and $\rho / L_{p}=1$, Eq. (35) leads to the requirement $I_{\text {aux }} \eta_{\text {aux }} / I_{\text {tor }} \cong 0.01$.

If this criterion is met, the island will not grow to the large saturated island width value given by Eq. (19), but instead grow to an island width comparable to the radial localization size of the auxiliary current. This small saturated island width is given by Eq. (22).

Even if complete stabilization of the large saturated island width cannot be achieved, the phased auxiliary current will still have a beneficial effect. As pointed out in the middle curve of Fig. 3, a nonlinear island width threshold is created if the added current is below the stabilization threshold. As mentioned previously, there are other mechanisms that have been invoked to explain a nonlinear magnetic island width threshold which are not included in this work. ${ }^{30-32,34,35}$ So at the very least, the added current drive can raise the threshold level.

Additionally, it has been pointed out that phasing the current source relative to the island width phase is not required to obtain a stabilizing effect. ${ }^{28}$ However, not phasing the current does reduce the efficiency as measured by $\eta_{\text {aux }}$. An exterior current source that is applied uniformly in $\alpha$ does introduce some phase localization do to the rapid equilibration of the current along the perturbed field lines. Mathematically, this is represented by the flux surface averaging procedure in the definition of the efficiency, Eq. (15). For the case of an externally applied unphased source, $\eta_{\text {aux }} \cong 0.37$ for $\delta_{J}<W .^{28}$

The effect of local heating on the neoclassical tearing mode has also been calculated. This effect introduces plasma currents in the island region through the self-consistent deformations of the Ohmic current profile. If the temperature profile peaks on the magnetic axis of the island relative to the separatrix, the currents are stabilizing. While this mechanism is not predicted to eliminate saturated steady solutions of neoclassical MHD tearing modes, local heating can reduce 
the size of the saturated island, see Eqs. (31) and (34). Unlike the local current drive case, there is only one steady state root to the island evolution equation in the $D_{n c}>0, \Delta^{\prime}<0$ limit. In the case of uniform heating in the island region, the effect of the heating is quantified by the factor $Y$ described in Eq. (32) and rewritten here,

$$
\mathrm{Y}=\frac{18}{m^{2}} \frac{\sqrt{\epsilon} \beta \theta}{s^{2}} \frac{\rho^{2}}{L_{T e} L_{p}} \frac{\left(S / \chi_{\perp}\right)_{\text {inside }}}{\left(S / \chi_{\perp}\right)_{\text {outside }}},
$$

where $L_{T e}=-T_{e}\left(d T_{e} / d \rho\right)$ is the equilibrium electron temperature gradient and the approximation $\mu_{o} J_{\|}=2 B_{\theta} / \rho$ is made. Additionally, the equilibrium electron temperature gradient is assumed to be given by $n\left(d T_{e} / d \rho\right)$ $=-\rho\left(S / \chi_{\perp}\right) / 2$ where $S$ and $\chi_{\perp}$ are the characteristic heating sources and diffusion coefficients of the equilibrium outside the island separatrix. The last factor in Eq. (36) is the ratio of the heating to diffusion rates inside and outside the island region. Using the same numbers as used after Eq. (35), the characteristic size of $Y$ for $m=2$ islands is

$$
\mathrm{Y}=2.3 \frac{\left(S / \chi_{\perp}\right)_{\text {inside }}}{\left(S / \chi_{\perp}\right)_{\text {outside }}}
$$

In order for local heating to be an effective means of controlling the saturated island width (which requires $Y \gg 1$ ), either the heating rate inside the island separatrix needs to be larger than the equilibrium rate or the cross-field diffusivity inside the island needs to be much smaller than the characteristic value outside the island.

\section{CONCLUSIONS}

The effects of localized current drive and heating magnetic island formation in tokamaks have been calculated. Similar techniques for the prevention of resistive tearing modes and disruption control have been proposed and studied previously. ${ }^{16-28}$ In this work, the prevention and stabilization of neoclassical tearing modes have been emphasized.

It is concluded that a phased spatially localized auxiliary current driven at the O-point of the magnetic island can be an effective way to control the neoclassical MHD tearing mode by reducing its saturated island width to a level comparable to the radial localization width of the auxiliary current. The amount of current needed is of the order of a few percent of the total equilibrium current. If the current is short of this amount, it still can have the beneficial effect of raising the nonlinear magnetic island width threshold. Below this threshold, neoclassically driven magnetic islands would be prohibited from forming. Local heating of the island region cannot completely eliminate the mode but it can reduce the saturated island width. However, in order for this to be an appreciable effect, rather dramatic differences in the heating rate or diffusion properties inside the island separatrix relative to outside the island are needed.

\section{ACKNOWLEDGMENTS}

The authors would like to thank L. E. Zakharov and M. N. Rosenbluth for useful comments concerning the presentation of this material.
The work was supported by United States Department of Energy Grants No. DE-FG02-86ER53218 and No. DEFG02- 92ER54139.

${ }^{1}$ R. Carrera, R. D. Hazeltine, and M. Kotschenreuther, Phys. Fluids 29, 899 (1986).

${ }^{2}$ See National Technical Information Service Department No. DE6008946 (W. X. Qu and J. D. Callen, University of Wisconsin Plasma Report No. UWPR 85-5, 1985). Copies may be ordered from the National Technical Information Service, Springfield, Virginia 22161.

${ }^{3}$ C. C. Hegna and J. D. Callen, Phys. Fluids B 4, 1855 (1992).

${ }^{4}$ C. C. Hegna, J. D. Callen, T. A. Gianakon, W. X. Qu, A. I. Smolyakov, and J. P. Wang, Plasma Phys. Controlled Fusion 35, 987 (1993).

${ }^{5}$ Z. Chang, J. D. Callen, C. C. Hegna, E. D. Fredrickson, R. V. Budny, K. M. McGuire, M. C. Zarnstorff, and the TFTR Group, Phys. Rev. Lett. 74, 4663 (1994).

${ }^{6}$ H. R. Wilson, M. Alexander, J. W. Connor, A. M. Edwards, D. Gates, O. Grüber, R. J. Hastie, C. C. Hegna, T. C. Hender, R. J. La Haye, L. L. Lao, A. W. Morris, C. M. Roach, E. J. Strait, T. S. Taylor, M. Valovic and H. Zohm, Plasma Phys. Controlled Fusion 38, A149 (1996).

${ }^{7}$ R. J. La Haye, J. D. Callen, M. S. Chu, S. Deshpande, T. A. Gianakon, C. C. Hegna, S. Jardin, L. L. Lao, J. Maniskam, D. A. Monticello, A. Pletzer, A. H. Reiman, O. Sauter, E. J. Strait, T. S. Taylor, A. D. Turnbull, and H. R. Wilson, "Practical beta limit in ITER-shaped discharges in DIII-D and its increase by higher collisionality", to appear in Plasma Physics and Controlled Nuclear Fusion Research 1996, 16th Conference Proceedings (International Atomic Energy Agency, Vienna, 1997) Paper AP1-21.

${ }^{8}$ O. Sauter, R. J. La Haye, Z. Chang, D. A. Gates, Y. Kamada, H. Zohm, A. Bondeson, D. Boucher, J. D. Callen, M. S. Chu, T. A. Gianakon, R. W. Harvey, C. C. Hegna, L. L. Lao, D. A. Monticello, F. Perkins, A. Pletzer, A. H. Reiman, M. Rosenbluth, E. J. Strait, T. S. Taylor, A. D. Turnbull, F. Waelbroeck, J. C. Wesley, H. R. Wilson, and R. Yoshino, Bull Am. Phys. Soc. 41, 1528 (1996); Phys. Plasmas 4, 1654 (1997).

${ }^{9}$ D. A. Gates, B. Lloyd, A. W. Morris, G. McArdle, M. O'Brien, M. Valovic, C. D. Warrick, H. R. Wilson, and the COMPASS-D and ECRH Teams, " $\beta$-Limit Studies and the Effect of Error Fields at Low Collisionality on Compass-D" to appear in Ref. 7, Paper AP1-17.

${ }^{10}$ Z. Chang, E. D. Fredrickson, J. D. Callen, K. M. McGuire, M. G. Bell, R. V. Budny, C. E. Bush, D. S. Darrow, A. C. Janos, L. C. Johnson, H. K. Park, S. D. Scott, J. D. Strachan, E. J. Synakowski, G. Taylor, R. M. Wieland, M. C. Zarnstorff, S. J. Zweben, and the TFTR Team, Nucl. Fusion 34, 1309 (1994).

${ }^{11}$ D. A. Monticello and R. White, Phys. Fluids 23, 367 (1980).

${ }^{12}$ M. S. Chu, H. Izeki, and T. H. Jenson, Phys. Fluids 27, 472 (1984).

${ }^{13}$ E. Lazzaro and M. F. F. Nave, Phys. Fluids 31, 1623 (1988).

${ }^{14}$ G. Bosia and E. Lazzaro, Nucl. Fusion 31, 1003 (1991).

${ }^{15}$ A. W. Morris, T. C. Hender, J. Hugill, P. S. Haynes, P. C. Johnson, B. Lloyd, D. C. Robinson, C. Silvester, A. Arshal, and G. M. Fishpool, Phys. Rev. Lett. 64, 1254 (1990).

${ }^{16}$ V. Chan and G. Guest, Nucl. Fusion 22, 272 (1982).

${ }^{17}$ Y. Yoshioka, S. Kinoshita, and T. Kobayashi, Nucl. Fusion 24, 565 (1984).

${ }^{18} \mathrm{P}$. H. Rebut and M. Hugon, in Plasma Physics and Controlled Nuclear Fusion Research 1984, (Proceedings of the 10th International Conference, London, 1984) (International Atomic Energy Agency, Vienna, 1985), Vol. 2, p. 197

${ }^{19}$ G. Kurita, T. Tuda, M. Azumi, T. Takizuka, and T. Takeda, Nucl. Fusion 34, 1497 (1994).

${ }^{20}$ V. V. Alikaev, Yu. I. Arsentiev, A. A. Bagdasarov, E. L. Berezovskij, and A. A. Bogshegovskij, in Ref. 18, Vol. 1, p. 419.

${ }^{21}$ The TFR Group, and the FOM/ECRH Team, Nucl. Fusion 28, 1995 (1988).

${ }^{22}$ K. Hoshino, M. Mori, T. Yamamoto, H. Tamai, T. Shoji, Y. Miura, H. Aikawa, S. Kasai, T. Kawakami, H. Kawashimi, M. Maeno, T. Matsuda, K. Oasa, K. Odajima, H. Ogawa, T. Ogawa, T. Seike, T. Shiina, K. Uehara, T. Yamauchiuu, N. Suzuki, and H. Maeda, Phys. Rev. Lett. 69, 2208 (1992). 
${ }^{23}$ D. C. Sing, M. E. Austin, D. L. Brower, J. Y. Chen, R. F. Gandy, C. X. Yu, X.-H. Wang, A. Bhattacharjee, and D. D. Schnack, Phys. Fluids B 5, 3239 (1993).

${ }^{24}$ A. Reiman, Phys. Fluids 26, 1338 (1983).

${ }^{25}$ P. H. Rutherford, in Basic Physical Processes of Toroidal Fusion Plasmas, (Proceedings of the Course and Workshop, Varenna, 1985) (Commission of the European Communities, Brussels, 1986), Vol. 2, p. 531.

${ }^{26}$ C. C. Hegna and A. Bhattacharjee, Phys. Rev. Lett. 63, 2056 (1989).

${ }^{27}$ C. C. Hegna and A. Bhattacharjee, Phys. Fluids B 2, 1804 (1990).

${ }^{28}$ F. W. Perkins and M. N. Rosenbluth (private communication).

${ }^{29}$ P. H. Rutherford, Phys. Fluids 16, 1903 (1973).
${ }^{30}$ R. Fitzpatrick, Phys. Plasmas 2, 825 (1995).

${ }^{31}$ N. N. Gorolenkov, R. V. Budny, Z. Chang, M. V. Gorelenkova, and L. E. Zakharov, Phys. Plasmas 3, 3379 (1996).

${ }^{32}$ T. A. Gianakon, J. D. Callen, and C. C. Hegna, Phys. Plasmas 3, 4637 (1996).

${ }^{33}$ C. C. Hegna and J. D. Callen, Phys. Fluids B 4, 4072 (1992).

${ }^{34}$ A. I. Smolyakov, Plasma Phys. Controlled Fusion 35, 657 (1993).

${ }^{35}$ H. R. Wilson, J. W. Connor, R. J. Hastie, and C. C. Hegna, Phys. Plasmas 3, 248 (1996).

${ }^{36}$ M. Kotschenreuther, R. D. Hazeltine, and P. J. Morrison, Phys. Fluids 28, 294 (1985). 